\title{
Dielectric Properties of PE/Clay Nanocomposites
}

\author{
E. David, ${ }^{1}$ M. Fréchette, ${ }^{2}$ B. Zazoum, ${ }^{1}$ C. Daran-Daneau, ${ }^{3}$ A. D. Ngô, ${ }^{1}$ and H. Couderc ${ }^{1}$ \\ ${ }^{1}$ École de Technologie Supérieure, Montréal, QC, Canada H3C 1 K3 \\ ${ }^{2}$ Institut de Recherche d'Hydro-Québec, Varennes, QC, Canada J3X 1S1 \\ ${ }^{3}$ Dessau, Montréal, QC, Canada H3B 4 V3 \\ Correspondence should be addressed to E. David; eric.david@etsmtl.ca
}

Received 4 February 2013; Revised 23 May 2013; Accepted 26 May 2013

Academic Editor: Alan K. T. Lau

Copyright (C) 2013 E. David et al. This is an open access article distributed under the Creative Commons Attribution License, which permits unrestricted use, distribution, and reproduction in any medium, provided the original work is properly cited.

\begin{abstract}
Polyethylene/nanoclay specimens containing from 0 to $5 \%$ nanoclays were prepared from a commercially available premixed $\mathrm{PE} /$ nanoclay masterbatch containing $50 \%$ wt of nanoclay. The masterbatch was diluted to the desired concentration by adding $\mathrm{PE}$ along with various amounts of compatibilizer in order to achieve the best possible dispersion of the nanoclay platelets. The dielectric response of the compounded samples was investigated using a combination of time and frequency-domain spectroscopy in order to cover a wide frequency window. Both techniques were in good agreement when the time-domain data was transformed into frequency-domain data. Despite their low concentration, the addition of the dispersed nanoclays led to a significant alteration of the material dielectric response in the form of the appearance of various interfacial relaxation processes and an increase of charge carrier transport within the insulation material. Moreover, an onset of nonlinear charge transport process was observed at moderate fields for specimens containing a relatively low level of nanoclays. The high-field breakdown strength was shown to have been improved by the incorporation of the nanoparticles, particularly when the exfoliation was enhanced by the use of a maleic anhydride grafted polyethylene compatibilizer.
\end{abstract}

\section{Introduction}

Certain properties of polymers have long been known to be favorably altered by a small addition of nanofillers [1-3]. Layered silicate-based polymer nanocomposites have attracted considerable interest because nanometric scale dispersion can be achieved through a combination of chemical and mechanical processes $[4,5]$, which result in high aspect ratio fillers. When compared with conventional polymer composites, such structures can enhance the material functional properties, resulting in a material upgrade at relatively moderate cost. Montmorillonite, a natural clay with an aspect ratio higher than 100, and the most common member of the smectite clay family, is a good candidate for use as reinforcement for polymer nanocomposites preparation. These types of polyolefin dielectric polymers have become a common subclass of "Nanodielectrics" [6]. Although they exhibit a low degree of self-assembly [7], the improved dielectrics properties thus obtained have established their potential for high-voltage applications [8-10]. The polyethylene-based inorganic nanocomposite is of particular scientific interest whereas this polymer is nonpolar. Compatibility between the adjunct and the matrix, or "miscibility" [11], is recognized as critical for the formation of a nanocomposite. Thus far, many improvements of the properties have been achieved, using inorganic particles, and an overview of their results was given by Fréchette [12]. Furthermore, although many details affecting the macroscopic properties are now known [13], considerably little work has been carried out examining the dielectric behavior of polymer nanocomposites involving nanoclays. Moreover, montmorillonite, a natural clay, is amply available at low cost - which is a good thing for industrial applications. With nanoclay, the properties and implications linked with the choice of an intercalant/compatibilizer have been reported to be dominant [14], and the change of its composite morphology is further complicated by its structure change. This change is also affected by the polymer processing method. Quality control of these types of nanocomposites is still deficient and requires further experimentation. Although one would expect the nanoclay-polymer interface to be 
dominant [15], here, the degree of intercalation/exfoliation [16] is an additional parameter often eliciting the formation of a micrometric-size microstructure. Furthermore, it must be recalled that the aspect ratio of nanoclay favors large interfaces involving the formation of a diffuse ionic layer. However, nanoclays often form tactoïds.

In this paper, $1 \%, 3 \%$, and 5\% LLDPE/clay nanocomposites were prepared by melt compounding from a commercially available masterbatch whose polymer-clay compatibilization was facilitated by surface compatibilization based on iondipole interaction, which expands the nanoclay platelets to the point where individual platelets can be separated from one another by mechanical shear during material processing. The dielectric response both in the time and in the frequency domains and the breakdown strength of thin films were measured, and the results are reported and discussed in this paper.

\section{Experimental Approach}

2.1. Sample Preparation. Linear low-density polyethylene (LLDPE), with a melt flow index (MFI) of $0.1 \mathrm{~g} / \mathrm{min}$ and a density of $0.917 \mathrm{~g} / \mathrm{cm}^{3}$, was used to dilute a commercially available masterbatch of LLDPE/clay composite with $50 \%$ organomodified Montmorillonite (O-MMT) to the desired concentrations. Maleic anhydride grafted linear lowdensity polyethylene (LLDPE-g-MA), having a density of $0.940 \mathrm{~g} / \mathrm{cm}^{3}$ and MFI of $2.5 \mathrm{~g} / \mathrm{min}$, was used as a compatibilizer. This material is referred to as M603 in this text.

The neat LLDPE, the masterbatch, and the compatibilizer were dried at $40^{\circ} \mathrm{C}$ in a vacuum oven for a minimum of 48 hours prior to extrusion. The melt compounding was achieved through an extrusion process using a corotating twin-screw extruder coupled with a metering feeder in order to control the feed rate. More details on the compounding process can be found elsewhere [17]. The pellets obtained after extrusion were press molded to form thin plates $(\sim 500 \mu \mathrm{m})$ using an electrically heated hydraulic press. The molding temperature and pressure were $178^{\circ} \mathrm{C}$ and $5 \mathrm{MPa}$, respectively. Table 1 gives a list of the different samples used, along with their composition, melting point, and crystallinity, as measured by Differential Scanning Calorimetry [17].

2.2. Dielectric Measurements. Frequency-domain measurements (FD) were conducted with a broadband dielectric spectrometer for a $10^{-3}$ to $10^{6} \mathrm{~Hz}$ frequency range in a temperature-controlled chamber. A $3 \mathrm{~V}$ sinusoidal voltage was applied across the sample. Time-domain measurements (TD) were conducted by applying a step voltage across the samples in a two-active electrode setup. Both the polarization and the depolarization current were continuously monitored for 9000 s. Voltage steps from 400 to $1000 \mathrm{~V}$ were used, creating a nearly uniform electrical field ranging from 0.7 to $1.8 \mathrm{kV} / \mathrm{mm}$.

2.3. Breakdown Measurements. The dielectric breakdown measurements were conducted according to the ASTM D14909 standard and using a BAUR DTA 100 device to hold both the samples and the surrounding media. The specimens were
TABLE 1: DSC results for pure LLDPE and LLDPE/O-MMT nanocomposites.

\begin{tabular}{lccc}
\hline $\begin{array}{l}\text { Sample } \\
\text { identification }\end{array}$ & Composition & Melting point & Cristallinity \\
\hline LLDPE & 100\% LLDPE & 107.7 & 33.7 \\
LLDPE/0/1 & $99 \%$ LLDPE & 108.0 & 36.8 \\
& 1\% O-MMT & & \\
LLDPE/0/3 & 97\% LLDPE & 107.1 & 35.3 \\
& 3\% O-MMT & & \\
& $92 \%$ LLDPE & & \\
LLDPE/5/3 & 5\% LLDPE-g-MA & & \\
& 3\% O-MMT & & \\
& 87\% LLDPE & 107.4 & \\
LLDPE/10/3 & 10\% LLDPE-g-MA & \\
& 3\% O-MMT & & \\
& 83\% LLDPE & & \\
LLDPE/15/3 & 15\% LLDPE-g-MA & 107.1 & \\
& 3\% O-MMT & & 34.0 \\
LLDPE/0/5 & 95\% LLDPE & 107.7 \\
\hline
\end{tabular}

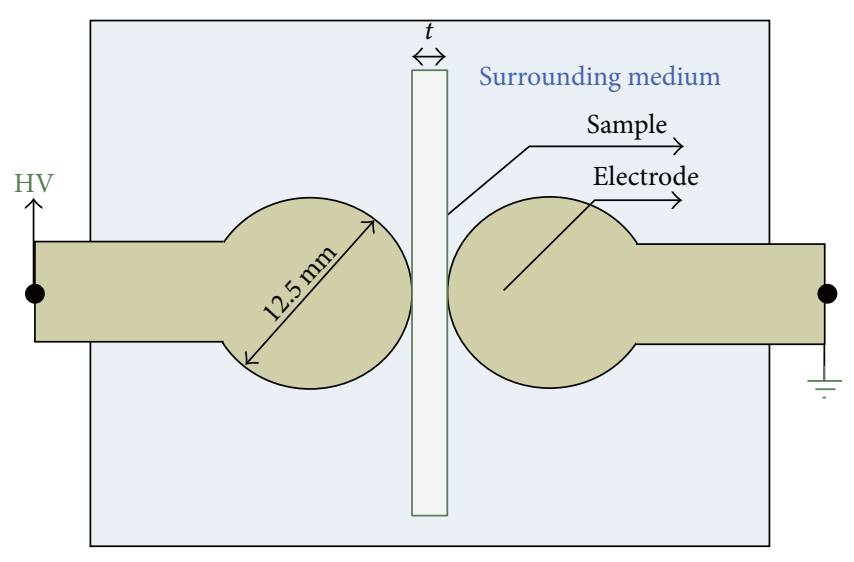

Figure 1: Dielectric breakdown measurement setup.

placed between two $12.7 \mathrm{~mm}$ hemispherical electrodes (type 5 ) and held by a small applied pressure. The whole setup was immerged in a surrounding medium, as shown in Figure 1. According to ASTM D149, the short-time test (Method A) was chosen and the rate-of-rise was set to $2 \mathrm{kV} / \mathrm{s}$ and going from zero until the sample failed. The failure criterion of the current-sensing element was set to $6 \mathrm{~mA}$.

The measurements were made using mineral oil (Voltesso 35, ESSO Imperial Oil) as a surrounding medium. The tested samples were labeled as listed in Table 1. To highlight the effect of thickness on the dielectric strength variation, the dielectric breakdown measurement was performed for two different thicknesses on some of the PE/clay nanocomposites. Between each sample, the electrodes were cleaned and the surrounding medium was removed and replaced with a completely new one. Each sample was composed of $n=20$ specimens. For each breakdown, the dielectric strength $E$ was calculated according to the breakdown voltage $V$ and 
TABLE 2: Dissipation factor at power frequency $(60 \mathrm{~Hz})$ for LLDPE/ nanoclay composites.

\begin{tabular}{lccc}
\hline & $20^{\circ} \mathrm{C}$ & $40^{\circ} \mathrm{C}$ & $60^{\circ} \mathrm{C}$ \\
\hline LLDPE & $3.59 \times 10^{-4}$ & $3.75 \times 10^{-4}$ & $9.74 \times 10^{-4}$ \\
LLDPE/0/1 & $8.10 \times 10^{-3}$ & $1.33 \times 10^{-2}$ & $8.81 \times 10^{-3}$ \\
LLDPE/0/3 & $2.14 \times 10^{-2}$ & $4.35 \times 10^{-2}$ & $3.59 \times 10^{-2}$ \\
LLDPE/10/3 & $5.12 \times 10^{-3}$ & $3.59 \times 10^{-2}$ & $4.49 \times 10^{-2}$ \\
LLDPE/0/5 & $3.88 \times 10^{-2}$ & $8.53 \times 10^{-2}$ & $8.88 \times 10^{-2}$ \\
\hline
\end{tabular}

the sample thickness $t$ at the breakdown point, according to the following equation:

$$
E=\frac{V}{t}
$$

Each dielectric strength value $E_{i}$ was then sorted in ascending order and associated with an index $i \in \llbracket 1, n \rrbracket$.

According to the IEC/IEEE 62539 standard (based on IEEE Standard 930-2004), the dielectric strength data were treated by using the two-parameter Weibull statistical distribution, where the cumulative distributive function of dielectric strength $P(E)$ can be written as

$$
P(E)=1-e^{-(E / \alpha)^{\beta}},
$$

where $\alpha$ is the scale factor representing the dielectric strength, with $P(\alpha)=0.632$, and where $\beta$ is the shape factor related to the data scattering.

For each sample, the cumulative probability of failure, $P(i, n)$, associated with the $E_{i}$ data was approximated by

$$
P(i, n) \approx \frac{i-0.44}{n+0.25} .
$$

The calculation of the estimator of the scale factor of the Weibull distribution for each set of data was done by assigning weightings corresponding to the White method as suggested in the IEC/IEEE 62539 standard. This estimator, as given in Table 2, will be considered as the material's characteristic breakdown strength. The confidence bounds given in Table 3 were also calculated according to the IEEE standard.

\section{Theoretical Background}

In the time domain, the behavior of a linear insulating system is characterized by its conductivity $\sigma$ and its dielectric response function. When a linear dielectric material is subjected to an arbitrarily time-varying voltage function, $U(t)$, the total current measured by an external circuit, $I(t)$, is given by $[18]$

$$
\begin{aligned}
I(t)= & \frac{C_{o} \sigma U(t)}{\varepsilon_{o}} \\
& +C_{o} \frac{\partial}{\partial t}\left[\varepsilon_{\infty} U(t)+\int_{0}^{\infty} f_{s}(\tau) U(t-\tau) d \tau\right],
\end{aligned}
$$

where $C_{o}$ is the vacuum capacitance, $\varepsilon_{\infty}$ is the "high frequency" relative permittivity, $\varepsilon_{o}$ is the vacuum permittivity,
TABLE 3: Breakdown strength of various PE/nanoclay composites.

\begin{tabular}{lcccc}
\hline Sample & $\begin{array}{c}\text { Average } \\
\text { thickness }(\mathrm{mm})\end{array}$ & $\begin{array}{c}\widehat{\alpha} \\
(\mathrm{kV} / \mathrm{mm})\end{array}$ & $\widehat{\beta}$ & $\begin{array}{c}\alpha_{l}-\alpha_{u} \\
(\mathrm{kV} / \mathrm{mm})\end{array}$ \\
\hline LLDPE-lab\#1 & 0.59 & 60.7 & 25.5 & $59.7-61.6$ \\
LLDPE-lab\#2 & 0.59 & 61.0 & 29.7 & $60.1-61.8$ \\
LLDPE-lab\#2 & 0.61 & 58.8 & 17.1 & $57.4-60.1$ \\
LLDPE-lab\#1 & 0.53 & 72.9 & 18.8 & $71.3-74.3$ \\
LLDPE/0/1-lab\#1 & 0.59 & 65.8 & 14.6 & $63.9-67.5$ \\
LLDPE/0/1-lab\#1 & 0.53 & 72.3 & 19.6 & $70.8-73.7$ \\
LLDPE/0/1-lab\#2 & 0.59 & 66.1 & 17.2 & $64.5-67.5$ \\
LLDPE/0/1-lab\#2 & 0.58 & 62.9 & 17.9 & $61.4-64.2$ \\
LLDPE/0/3-lab\#1 & 0.58 & 69.6 & 19.7 & $68.1-70.9$ \\
LLDPE/0/3-lab\#1 & 0.53 & 72.1 & 19.3 & $70.6-73.5$ \\
LLDPE/0/3-lab\#2 & 0.58 & 69.8 & 18.1 & $68.2-71.2$ \\
LLDPE/0/3-lab\#2 & 0.57 & 66.6 & 18.2 & $65.1-68.0$ \\
LLDPE/10/3-lab\#2 & 0.56 & 69.6 & 17.9 & $68.0-71.1$ \\
\hline
\end{tabular}

and $f_{s}(t)$ is the "slow" dielectric response function. The Fourier transform of (4) leads to the frequency-domain expression of the complex permittivity [18]. When a step voltage is applied across the dielectric material during a charging time $t_{c}$, the voltage function is given by

$$
U(t)= \begin{cases}0 & t<0 \\ U_{o} & 0 \leq t \leq t_{c} \\ 0 & t>t_{c}\end{cases}
$$

which leads to charge and discharge currents given by

$$
I(t)=\left\{\begin{array}{l}
\frac{C_{o} \sigma}{\varepsilon_{o}} U_{o}+U_{o} C_{o}\left[\varepsilon_{\infty} \delta(t)+f_{s}(t)\right], \\
-U_{o} C_{o}\left[\varepsilon_{\infty} \delta\left(t-t_{c}\right)+f_{s}\left(t-t_{c}\right)-f(t)\right], \\
t>t_{c} .
\end{array}\right.
$$

The last term of the second equation in (6), the socalled memory term, vanishes for an infinite charging time (assuming a monotonically decreasing response function). Time domain data can be converted into frequency-domain data using various methods, including a numerical Fourier transform, by fitting an expression for the dielectric response function that can be subsequently analytically transformed [19], or through the Hamon approximation [20]. By using to fit the discharge current, an expression such as [19]

$$
f_{s}(t)=A\left[e^{-t / \tau}\left(\frac{t}{\tau}\right)^{-m}+\left(1-e^{-t / \tau}\right)\left(\frac{t}{\tau}\right)^{-n}\right],
$$

it allows the ready calculation of the complex dielectric susceptibility since the Fourier transform of expression (7) has an analytical solution [19]. For weakly dispersive materials, the Hamon approximation can also be used to extract the imaginary permittivity as a function of frequency from the charge or the discharge currents, using the following relation:

$$
\varepsilon^{\prime \prime}(f) \approx \frac{i(0.1 / f)}{2 \pi f C_{o} U_{o}} .
$$


By definition, the effective dielectric constant of a heterogeneous linear material can be defined by [21]

$$
\langle\widehat{D}\rangle=\varepsilon_{o} \widehat{\varepsilon}_{c}\langle\widehat{E}\rangle,
$$

where $\widehat{D}$ and $\widehat{E}$ are, respectively, the phasor representing the dielectric displacement and the electric field vectors, $\widehat{\varepsilon}_{c}$ is the complex dielectric function, and the brackets denote an average over the material's volume. For two-component dielectric media, it leads to

$$
\widehat{\varepsilon}_{c}=\frac{q_{1} \widehat{\varepsilon}_{1}\left\langle\widehat{E}_{1}\right\rangle+q_{2} \widehat{\varepsilon}_{2}\left\langle\widehat{E}_{2}\right\rangle}{\langle\widehat{E}\rangle},
$$

where $q_{1}$ and $q_{2}$ are the volume fractions of phase 1 and phase 2. The average electrical field is obviously given by

$$
\langle\widehat{E}\rangle=q_{1}\left\langle\widehat{E}_{1}\right\rangle+q_{2}\left\langle\widehat{E}_{2}\right\rangle .
$$

An analytical calculation of the electrical field in a composite material can only be made if the minority phase is present in a small concentration and for regular shape inclusions. A number of results can be found where an exact solution for several matrix systems with periodic arrangements of regular inclusions is obtained [22,23]. In the case of a dilute suspension of ellipsoidal shape inclusions with a complex permittivity $\widehat{\varepsilon}_{2}$ in a continuum matrix of complex permittivity $\widehat{\varepsilon}_{1}$, it is possible to use the solution of the single-inclusion problem, which gives the following expression for the field ratio inside and outside the inclusion [24]:

$$
\frac{\left\langle\widehat{E}_{2}\right\rangle}{\left\langle\widehat{E}_{1}\right\rangle}=\sum_{i=1}^{3} \frac{\cos ^{2}\left(\alpha_{i}\right)}{1+\left(\widehat{\varepsilon}_{2} / \widehat{\varepsilon}_{1}-1\right) A_{i}},
$$

where the $\alpha_{i}$ is the angles between the applied field and the $i$ th axis of the ellipsoid and $A_{i}$ is its depolarization factor [24]. For randomly oriented ellipsoidal particles, $\cos ^{2}\left(\alpha_{1}\right)=$ $\cos ^{2}\left(\alpha_{2}\right)=\cos ^{2}\left(\alpha_{3}\right)=1 / 3$. For spherical particles, $A_{1}=$ $A_{2}=A_{3}=1 / 3$. In the case of spheroids for which two axes are equal $(a=b \neq c)$, the analytical expressions for $A_{i}$ for oblate spheroids (disk-like spheroids with $a=b>c$ ) and prolate spheroids (needle-like spheroids with $a=b<c$ ) can be found in the literature [24]. In the case of a spherical void $\left(\varepsilon_{2}=1\right)$ inside a dielectric medium with a permittivity $\varepsilon_{1}$, (12) leads to the well-known result expressing the field reinforcement inside a cavity:

$$
\frac{\left\langle\widehat{E}_{2}\right\rangle}{\left\langle\widehat{E}_{1}\right\rangle}=\frac{3 \varepsilon_{1}}{2 \varepsilon_{2}+1} .
$$

Combining (10), (11), and (12), we obtain

$$
\begin{aligned}
\widehat{\varepsilon}_{c}= & \left(\widehat{\varepsilon}_{1}\left(1-q_{2}\right)+\widehat{\varepsilon}_{2} q_{2} \sum_{i=1}^{3} \frac{\cos ^{2}\left(\alpha_{i}\right)}{1+\left(\widehat{\varepsilon}_{2} / \widehat{\varepsilon}_{1}-1\right) A_{i}}\right) \\
& \times\left(1-q_{2}+q_{2} \sum_{i=1}^{3} \frac{\cos ^{2}\left(\alpha_{i}\right)}{1+\left(\widehat{\varepsilon}_{2} / \widehat{\varepsilon}_{1}-1\right) A_{i}}\right)^{-1},
\end{aligned}
$$

which leads to

$$
\begin{aligned}
\widehat{\varepsilon}_{c}= & \left(\widehat{\varepsilon}_{1}\left(1-q_{2}\right)+\frac{\widehat{\varepsilon}_{2} q_{2}}{3} \sum_{i=1}^{3} \frac{\widehat{\varepsilon}_{1}}{\widehat{\varepsilon}_{1}+\left(\widehat{\varepsilon}_{2}-\widehat{\varepsilon}_{1}\right) A_{i}}\right), \\
& \times\left(1-q_{2}+\frac{q_{2}}{3} \sum_{i=1}^{3} \frac{\widehat{\varepsilon}_{1}}{\widehat{\varepsilon}_{1}+\left(\widehat{\varepsilon}_{2}-\widehat{\varepsilon}_{1}\right) A_{i}}\right)^{-1},
\end{aligned}
$$

for randomly oriented inclusions. This method is known as the effective-medium or the Maxwell approximation. For spherical inclusions, it leads to the well-known MaxwellGarnett equation [25]. Since this approach does not take into account the details of microstructural information, it cannot provide an accurate quantitative prediction of $\mathrm{PE} /$ clay nanocomposites that usually exhibit a very complex microstructure. However, this approximation can provide qualitative trends on the dielectric response of a composite material that are useful for designing the material structure for a specific dielectric response. If filler particles, whose dielectric properties can be described simply by a frequencyindependent permittivity $\varepsilon_{f}$ and a conductivity $\sigma_{f}$, are perfectly oriented with their $c$ in the direction of the electrical field line $\left(\cos ^{2}\left(\alpha_{1}\right)=\cos ^{2}\left(\alpha_{2}\right)=0, \cos ^{2}\left(\alpha_{3}\right)=1\right)$ and dispersed in a nonconductive matrix with a frequency-independent dielectric constant $\varepsilon_{m}$, (11) reduces to a Maxwell-Wagner-Sillars equation [26], which leads to a single relaxation process whose complex permittivity is given by the following expression:

$$
\begin{gathered}
\widehat{\mathcal{\varepsilon}}_{c}=\varepsilon_{\infty}+\frac{\Delta \varepsilon}{1+j \omega \tau}, \\
\tau=\frac{\varepsilon_{o}\left(\varepsilon_{m}+A(1-q)\left[\varepsilon_{f}-\varepsilon_{m}\right]\right)}{A \sigma_{f}(1-q)},
\end{gathered}
$$

where $A$ and $q$ are, respectively, the shape factor or the depolarization factor along the $\mathrm{c}$ axis and the volume fraction of the minority phase. The expressions $\varepsilon_{\infty}$ and $\Delta \varepsilon$ as a function of $\varepsilon_{m}, \varepsilon_{f}, \sigma_{f}$, and $A$ can be found in the literature [26]. According to (16), the frequency of the relaxation peak, $2 \pi / \tau$, is strongly dependent upon the shape factor. Oblate spheroids with small axes parallel to the electric field have a higher relaxation frequency but a lower dielectric strength $\Delta \varepsilon$ than prolate spheroids. Accordingly, the exfoliation of the clay platelets is expected to lead to a lowering of the relaxation frequency since the individual platelet aspect ratio is around 200 [5]. In practice, it is not possible to fit the experimental data with a single relaxation time. To take into account the large variations in thickness of the various clay tactoïds dispersed within the polymeric material (or the dispersion of the shape factor), a broad relaxation peak, as expressed by the empirical Havriliak-Negami function [27], can be used, leading to the following equation for the complex permittivity:

$$
\widehat{\varepsilon}_{\mathrm{HN}}(\omega)=\varepsilon_{\infty}+\frac{\Delta \varepsilon}{\left[1+\left(j \omega \tau_{\mathrm{HN}}\right)^{\alpha_{\mathrm{HN}}}\right]^{\beta_{\mathrm{HN}}}},
$$




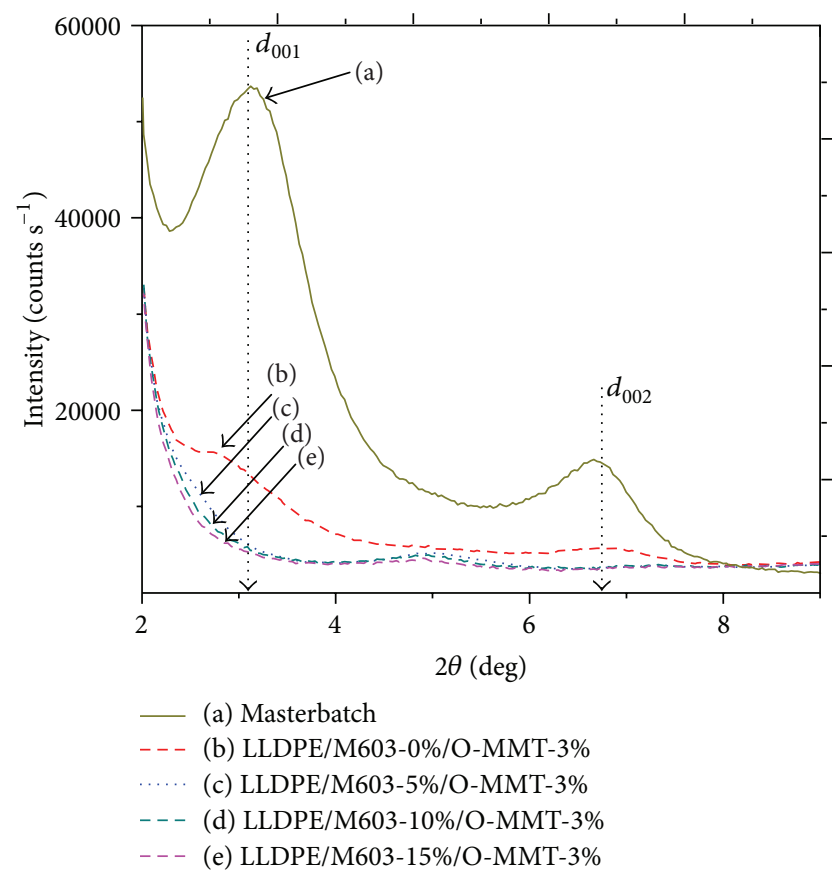

Figure 2: X-ray diffractograms of various LLDPE/M603/O-MMT nanocomposites.

in which $\varepsilon_{\infty}$ is the unrelaxed dielectric permittivity or the high frequency permittivity, $\Delta \varepsilon$ is the dielectric relaxation strength, $\tau_{\mathrm{HN}}$ is the relaxation time, and $\alpha_{\mathrm{HN}}$ and $\beta_{\mathrm{HN}}$ are the Havriliak-Negami parameters, respectively, describing the symmetric and asymmetric broadening of the relaxation time distribution function. In addition to interfacial relaxation peaks and dipolar relaxation peaks proper to each phase of a composite material, the contribution of the fluctuation of charge carriers will generally be superimposed on the other mechanisms. In the most general case, this contribution can be expressed by

$$
\widehat{\varepsilon}_{c c}(\omega)=B(j \omega)^{n-1}-j \frac{\sigma_{o}}{\omega \varepsilon_{o}},
$$

where $B$ and $n$ are material-dependent constants and $\sigma_{o}$ is the pure direct current conductivity, usually originating from the electronic conductivity.

\section{Results and Discussion}

4.1. Material Characterization. In this study, clay dispersion was mostly analyzed using X-ray diffractometery, Scanning Electronic Microscopy (SEM), Thermogravimetric Analysis (TGA) [6], and Transmission Electronic Microscopy (TEM). Figure 2 shows the X-ray diffraction spectra of the O-MMT masterbatch along with LLDPE/M603/O-MMT composites whose compatibilizer M603 concentration was changed from 0 to $15 \mathrm{wt} \%$. The 001 diffraction peak for the O-MMT masterbatch is located at about $2 \theta=3.2^{\circ}$, corresponding to a $d_{001}$ value of $2.77 \mathrm{~nm}$, which is probably very close to the basal spacing of the pure organomodified montmorillonite [4]. Therefore, not much intercalation was observed in the case

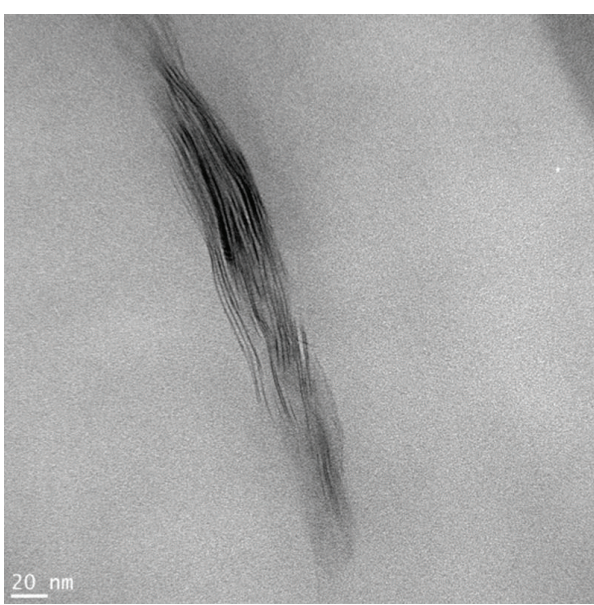

(a)

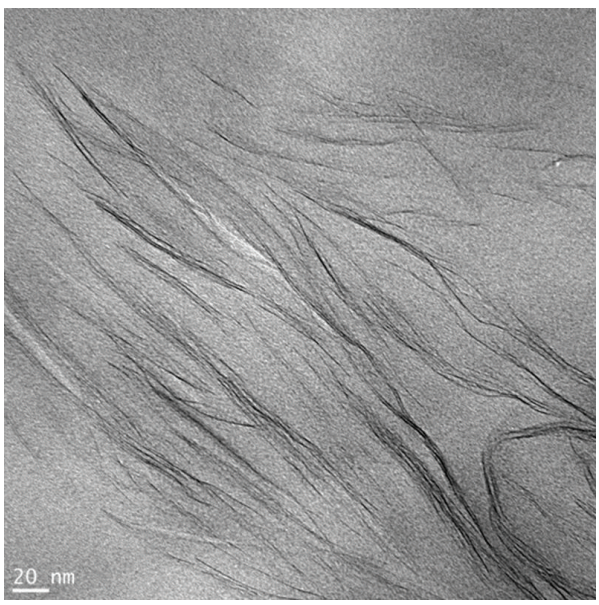

(b)

Figure 3: TEM pictures of (a) LLDPE/0/3 and (b) LLDPE/10/3 samples.

of the masterbatch, which can be viewed as a conventional microcomposite. However, LLDPE/O-MMT and LLDPE/ M603/O-MMT nanocomposites were found to be intercalated with the basal peak shifting to smaller angles, suggesting an increase in the gallery spacing, as shown in Figure 2. For the ternary nanocomposites LLDPE/M603/O-MMT with weight percentages of $87 / 10 / 3$ and $82 / 15 / 3$, curves (d) and (e), the disappearance of the $d_{001}$ peak shows that the nanoclay platelets start to separate from one another in the polymer matrix, suggesting at least a semiexfoliation of the platelets.

TEM images were obtained using a JEOL JEM-2100F microscope operating at a $200 \mathrm{kV}$ accelerating voltage. The TEM samples were cut to thin sections (50-80 nm thickness) at $-120^{\circ} \mathrm{C}$, using an ultramicrotome with diamond knife. Figure 3 shows the TEM picture of a stack of clay platelets for the (a) LLDPE/0/3 and the (b) LLDPE/10/3 samples. Obviously, the use of a compatibilizer significantly improves the intercalation of the clay layers. However, the clay platelets are not uniformly exfoliated within the polymeric matrix. Figure 4 presents a very large scale TEM picture of the LLDPE/10/3, showing the distribution of tactoids within 


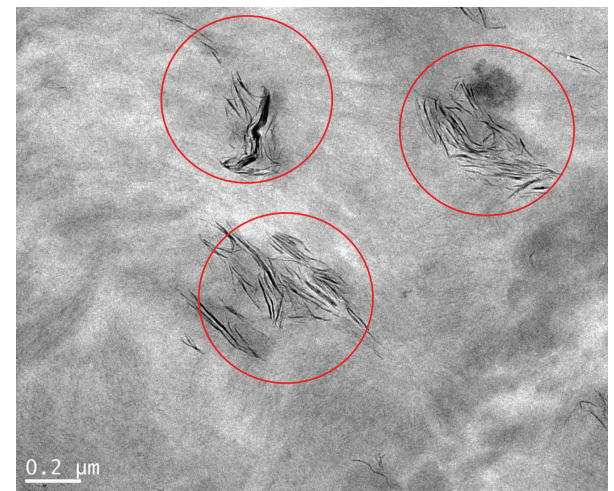

FIGURE 4: TEM picture of a LLDPE/10/3 specimen.
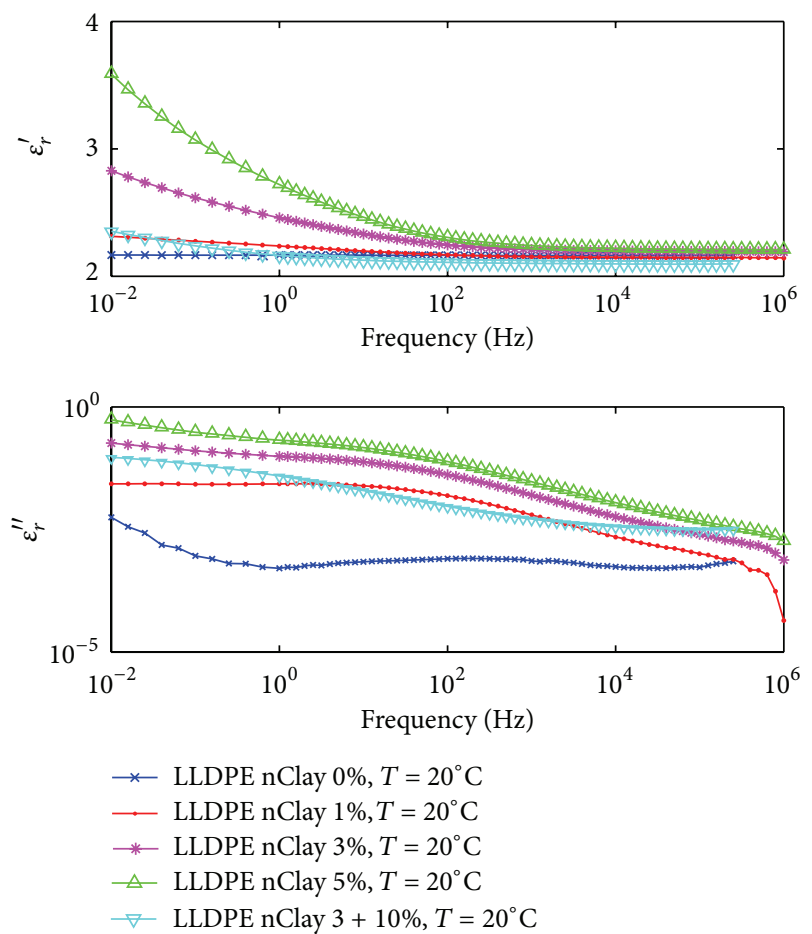

FIGURE 5: Real and imaginary parts of the complex permittivity of LLDPE and LLDPE/clay nanocomposites at $20^{\circ} \mathrm{C}$.

the PE matrix. This particular microstructure is expected to have a significant impact on the material dielectric response. This microstructure can be viewed as spherical inclusion of $\mathrm{PE} /$ clay with a high clay content dispersed in a pure $\mathrm{PE}$ matrix. In the case of the microstructure shown in Figure 4, the volume fractions would be approximately $q_{2}^{\prime}=0.06$ and $q_{2}^{\prime \prime}=0.2$, representing, respectively, the volume fraction of the clay platelets within the spherical inclusion (circles in Figure 4) and the volume fraction of these spherical inclusion within the PE matrix and the total volume fraction of clay being given by the product of $q_{2}^{\prime}$ and $q_{2}^{\prime \prime}$.

4.2. Dielectric Response of LLDPE/Clay Nanocomposites. Figure 5 shows the dielectric response of 100/0/0, 99/0/1,

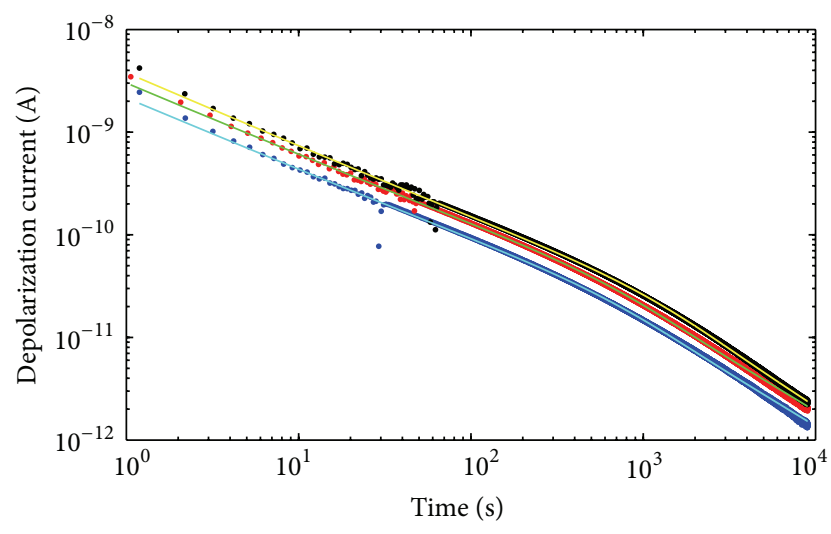

- Depolarization current $400 \mathrm{~V}$ — Analytic fitting curve $400 \mathrm{~V}$

- Depolarization current $600 \mathrm{~V}$ — Analytic fitting curve $600 \mathrm{~V}$

- Depolarization current $800 \mathrm{~V}$ — Analytic fitting curve $800 \mathrm{~V}$

(a)

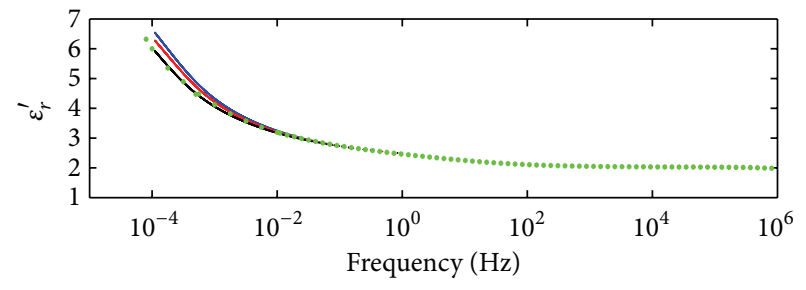

. TD spectroscopy and analytical transformation $U_{\mathrm{DC}}=400 \mathrm{~V}$

- TD spectroscopy and analytical transformation $U_{\mathrm{DC}}=600 \mathrm{~V}$

- TD spectroscopy and analytical transformation $U_{\mathrm{DC}}=800 \mathrm{~V}$

FD spectroscopy

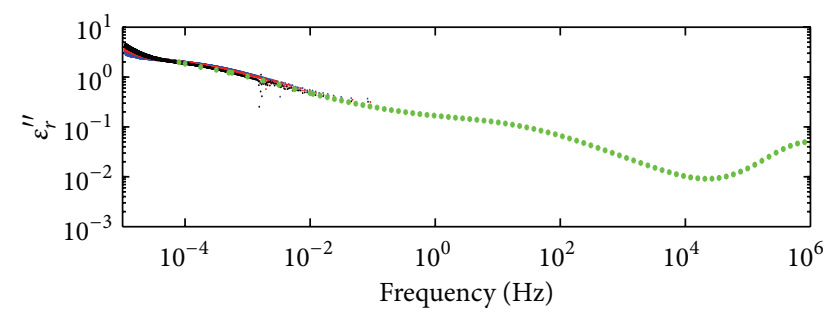

- TD spectroscopy and Hamon transformation $U_{\mathrm{DC}}=400 \mathrm{~V}$

- TD spectroscopy and Hamon transformation $U_{\mathrm{DC}}=600 \mathrm{~V}$

- TD spectroscopy and Hamon transformation $U_{\mathrm{DC}}=800 \mathrm{~V}$

- FD spectroscopy

(b)

FIgURE 6: Real and imaginary parts of the complex permittivity of a 5\% wt LLDPE and LLDPE/clay nanocomposites at $23^{\circ} \mathrm{C}$ : (a) time domain results; (b) combined time and frequency domain results.

97/0/3, 95/0/5 wt\% LLDPE/O-MMT nanocomposites, and a 87/10/3 wt\% LLDPE/M603/O-MMT nanocomposite obtained using frequency-domain (FD) dielectric spectroscopy in the $10^{-2}$ to $10^{6} \mathrm{~Hz}$ frequency range. Figure 6(b) shows the extended dielectric response from $10^{-4}$ to $10^{4} \mathrm{~Hz}$ for the 95/5 wt\% LLDPE/O-MMT nanocomposite. The curves for the real and the imaginary parts of the permittivity were 
obtained from both FD and TD dielectric spectroscopy. Figure 6(a) shows the discharge currents for electrical fields of $0.7,1.0$ and $1.4 \mathrm{kV} / \mathrm{mm}$, and the fittings with (7), allowing the computation of the real part of the permittivity, as shown in Figure 6(b). The imaginary part of Figure 6(b) was obtained by combining the Hamon approximation (see (8)) from the charge current and the FD dielectric measurements.

At $20^{\circ} \mathrm{C}$, the dielectric responses of the 1,3 , and $5 \mathrm{wt} \%$ LLDPE/O-MMT nanocomposites are somewhat similar, showing a broad interfacial relaxation peak around $10^{2} \mathrm{~Hz}$ and an increase of both real and imaginary permittivity toward low frequencies. This behavior is known as low frequency dispersion, which in this case, is most probably related to ionic conductivity leading to electrode polarization. Extending the measurements down to $10^{-5} \mathrm{~Hz}$ allows an observation of a second relaxation peak in the vicinity of $10^{-3} \mathrm{~Hz}$, as shown in Figure 6(b). This suggests that the broad interfacial relaxation peaks for the 1,3 , and $5 \mathrm{wt} \%$ LLDPE/OMMT nanocomposites are related to the random orientation of slightly intercalated stacks of platelets. This is in good agreement with the experimental results showing that the addition of $10 \%$ of compatibilizer significantly changes the dielectric response, leading to a shift of the main relaxation toward lower frequencies.

Figure 7 shows the FD dielectric responses for the 97/0/ $3 \mathrm{wt} \%$ LLDPE/O-MMT nanocomposite and the $87 / 10 / 3 \mathrm{wt} \%$ LLDPE/M603/O-MMT nanocomposite for temperatures of $0,20,40$, and $60^{\circ} \mathrm{C}$. As can be expected, the main interfacial relaxation peak shifts towards higher frequencies as the temperature increases, since the ionic conductivity along clay platelets increases with temperature, thus decreasing the relaxation time, as expressed in (16). Similarly to what can be seen in Figure 5, the ternary nanocomposite LLDPE/M603/O-MMT, with a weight percentage of 87/10/3, shows a similar dielectric behavior as its binary counterpart, with its interfacial relaxation peak shifted towards lower frequencies.

To relate the $\mathrm{PE} /$ clay nanocomposites dielectric response to their microstructure, the mean field theory (see (15)) can be used as a first approximation to obtain some insight on the expected dielectric response. Figure 8(a) shows the experimental imaginary permittivity for the $97 / 0 / 3$ and the $87 / 10 / 3$ binary and ternary nanocomposites at $20^{\circ} \mathrm{C}$ and the theoretical prediction of (15) for a spheroidal oblate filler with two different aspect ratios, $c / b=0.2$ and $c / b=0.05$. The complex permittivity of the clay fillers was simply assumed to be given by the following relation:

$$
\widehat{\varepsilon}_{2}(\omega)=\varepsilon_{\infty}-j \frac{\sigma_{o}}{\omega \varepsilon_{o}},
$$

with $\varepsilon_{\infty}=10, \sigma_{o}=1 \times 10^{-9} \mathrm{~S} / \mathrm{m}$, and $q_{2}=0.012$. The experimental data were used for the complex permittivity of the matrix, $\widehat{\varepsilon}_{1}$, in (15). As can be seen in Figure 8(a), the shift of the interfacial peak towards the low frequencies can be qualitatively explained, but the theoretical prediction does not provide a good fit with the experimental measurements. To improve the theoretical prediction, the detailed microstructure, as shown in Figure 4, must be taken into
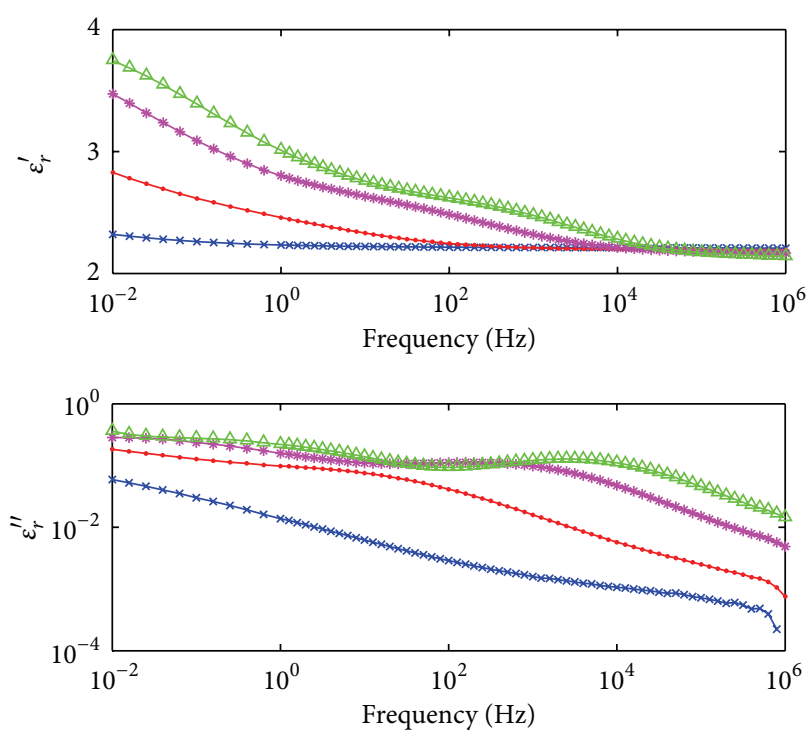

$\leftarrow$ LLDPE nClay $3 \%, T=0^{\circ} \mathrm{C}$

- LLDPE nClay $3 \%, T=20^{\circ} \mathrm{C}$

* LLDPE nClay $3 \%, T=40^{\circ} \mathrm{C}$

$\triangle$ LLDPE nClay $3 \%, T=60^{\circ} \mathrm{C}$

(a)
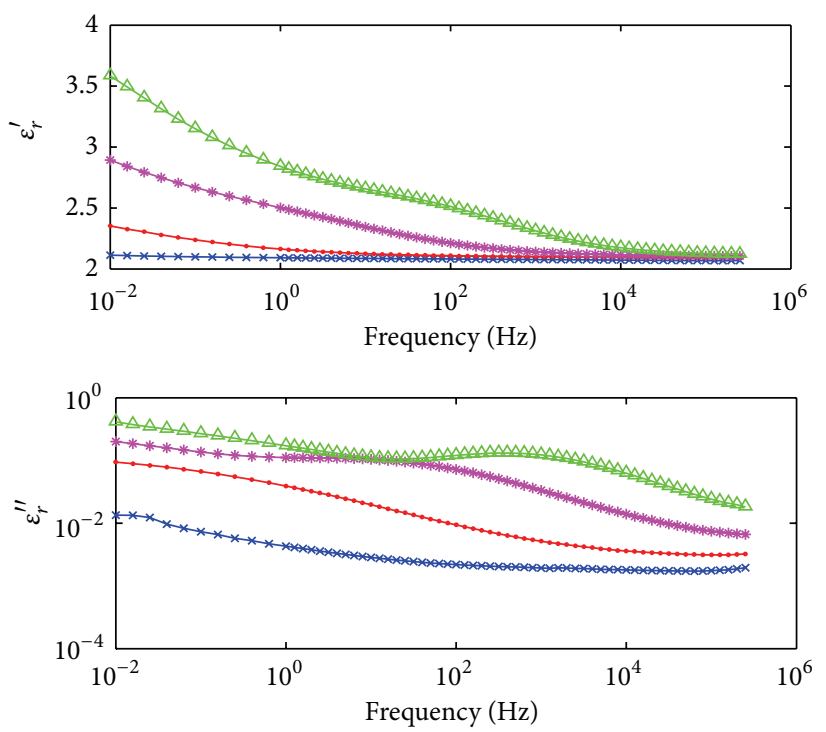

$\rightarrow$ LLDPE nClay $3+10 \%, T=0^{\circ} \mathrm{C}$
$\rightarrow$ LLDPE nClay $3+10 \%, T=20^{\circ} \mathrm{C}$
$*$ LLDPE nClay $3+10 \%, T=40^{\circ} \mathrm{C}$
$\triangle \quad$ LLDPE nClay $3+10 \%, T=60^{\circ} \mathrm{C}$

(b)

FIGURE 7: Real and imaginary parts of the complex permittivity for a (a) $3 \mathrm{wt} \%$ LLDPE/clay nanocomposite and a (b) $3 \mathrm{wt} \%$ LLDPE/clay nanocomposite with $10 \%$ of M603 compatibilizer for temperatures from 0 to $60^{\circ} \mathrm{C}$.

account. First, the complex permittivity of more a highlyloaded phase is computed. This phase is approximately shown in Figure 4 by the circles and is considered as a $\mathrm{PE} / \mathrm{clay}$ composite with a volume fraction of 0.2 of randomly oriented 


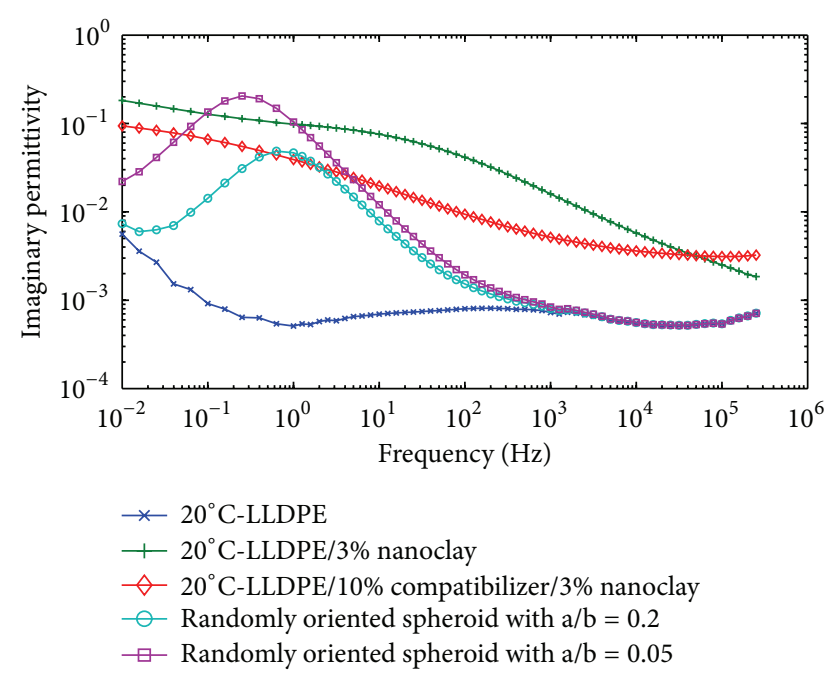

(a)

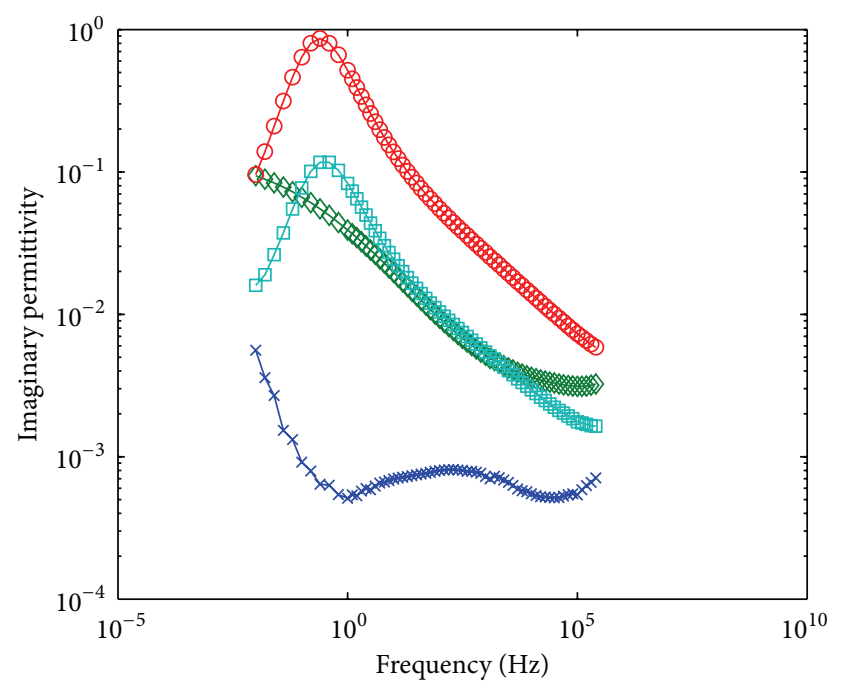

$-\approx 20^{\circ} \mathrm{C}$-LLDPE
$\neg 20^{\circ} \mathrm{C}-$ LLDPE$/ 10 \%$ compatibilizer $/ 3 \%$ nanoclay
- Randomly oriented spheroid with $\mathrm{a} / \mathrm{b}=0.05$
$\square$ Spherical inclusions

(b)

FIGURE 8: Imaginary part of the complex permittivity at $20^{\circ} \mathrm{C}$ for: a (a) $3 \mathrm{wt} \%$ LLDPE/clay nanocomposite and a $3 \mathrm{wt} \% \mathrm{LLDPE} / \mathrm{clay}$ nanocomposite with $10 \%$ of M603 compatibilizer compared with theoretical prediction from (15); (b) $3 \mathrm{wt} \%$ LLDPE/clay nanocomposite with $10 \%$ of M603 compatibilizer compared with a theoretical prediction obtained from using (15) two times.

spheroidal oblates having an aspect ratio of 0.05 . The dielectric properties of the clay tactoïds were then assumed to be given by the more realistic expression:

$$
\widehat{\varepsilon}_{2}(\omega)=\varepsilon_{\infty}+B(j \omega)^{n-1}-j \frac{\sigma_{o}}{\omega \varepsilon_{o}}
$$

which does take into account both the electronic (last term) and the ionic (second term) conductivities. Using the following numerical values:

$$
n=0.7, \quad B=25, \quad \varepsilon_{\infty}=10, \quad \sigma_{o}=1 \times 10^{-9} \mathrm{~S} / \mathrm{m},
$$

equation (15) yields to the curve labeled by the round markers in Figure $8(\mathrm{~b})$. When this computed complex permittivity is then considered as the reinforcing phase for spherical inclusions with a volume ratio of $0.06,(15)$ finally leads to the curve labeled by the square markers in Figure 8(b), which gives a reasonable fit with the experimental values.

An alternative way to describe the broad relaxation peak observed in the experimental data is in terms of a set of Debye functions with a continuous relaxation time distribution. The relaxation time distribution function, $L(\tau)$, can be evaluated numerically from the experimental data or calculated analytically from the parameters obtained from the fit with the empirical Havriliak-Negami function given in (17). Indeed, a fitting of the unknown parameters in (17) and (18) can be obtained using a numerical technique such as Levenberg-Marquardt iterations (the direct conductivity, $\sigma_{o}$, was assumed to be negligible). Figure 9(a) shows the dielectric loss $\varepsilon^{\prime \prime}$ versus the frequency for the $87 / 10 / 3$ nanocomposite at 40 and $60^{\circ} \mathrm{C}$. The solid lines are the fittings of the experimental data with the superposition of (17) and (18). Figure 9(b) illustrates the calculated relaxation time distribution function for these two temperatures. The values of the relaxation times of the main relaxation peak, $\tau_{\mathrm{HN}}$, were $1.10 \times 10^{-2}$ and $2.73 \times 10^{-4} \mathrm{~s}$ for sample $87 / 10 / 3$ at 40 and $60^{\circ} \mathrm{C}$, respectively. For the sample without a compatibilizer, the $97 / 0 / 3$ nanocomposite, two relaxation processes were clearly observed at 40 and $60^{\circ} \mathrm{C}$ in the dielectric response. This type of dielectric response is similar to what was also reported elsewhere for PE/clay nanocomposites [28, 29]. Figure 10 shows the experimental measurement and the fitting resulting from the use of (17) and (18), still with $\sigma_{o}=0$, and assuming two $\mathrm{H}-\mathrm{N}$ relaxation processes. The values of the relaxation times were $3.56 \times 10^{-4}$ and $2.63 \mathrm{~s}$ at $40^{\circ} \mathrm{C}$ and $5.26 \times 10^{-5}$ and $0.386 \mathrm{~s}$ at $60^{\circ} \mathrm{C}$.

For a possible future application as groundwall insulation in power cables, only the dielectric response at power frequencies $(60 \mathrm{~Hz}$ in North America) really matters. Table 2 below gives the dissipation factor, defined as the ratio of the imaginary and the real permittivities, at power frequencies for operating temperatures of 20,40 , and $60^{\circ} \mathrm{C}$, for different $\mathrm{PE} /$ clay nanocomposites. Similar values were reported in [28] for which the dissipation factor for a $4 \%$ wt PE/clay nanocomposite was found to be in the vicinity of $10^{-2}$. Despite the tremendous increase of the dielectric loss due to the addition of nanoclays (two orders of magnitude), the dielectric losses still remain low enough to cause no concern for power cable applications. Indeed, for a typical distribution cable of inner radius of $12 \mathrm{~mm}$, outer radius of $19 \mathrm{~mm}$, and operating at a phase-to-ground voltage of $14.4 \mathrm{kV}$, the dielectric loss for a dissipation factor of $10^{-2}$ would be less than $1 \mathrm{~W} / \mathrm{m}$, that is, at least of order of magnitude less than the losses due to the Joule heating of the conductor at full load. 


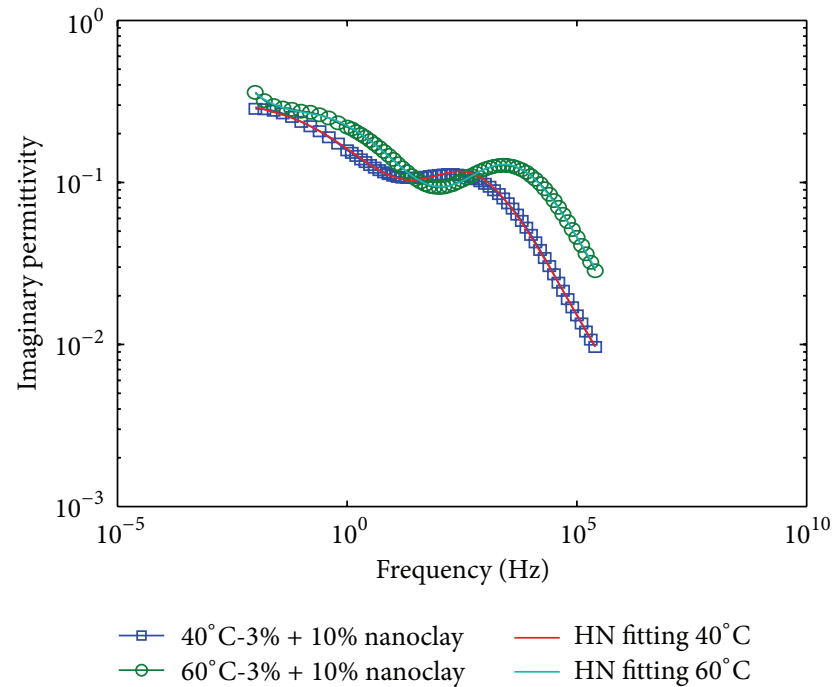

(a)

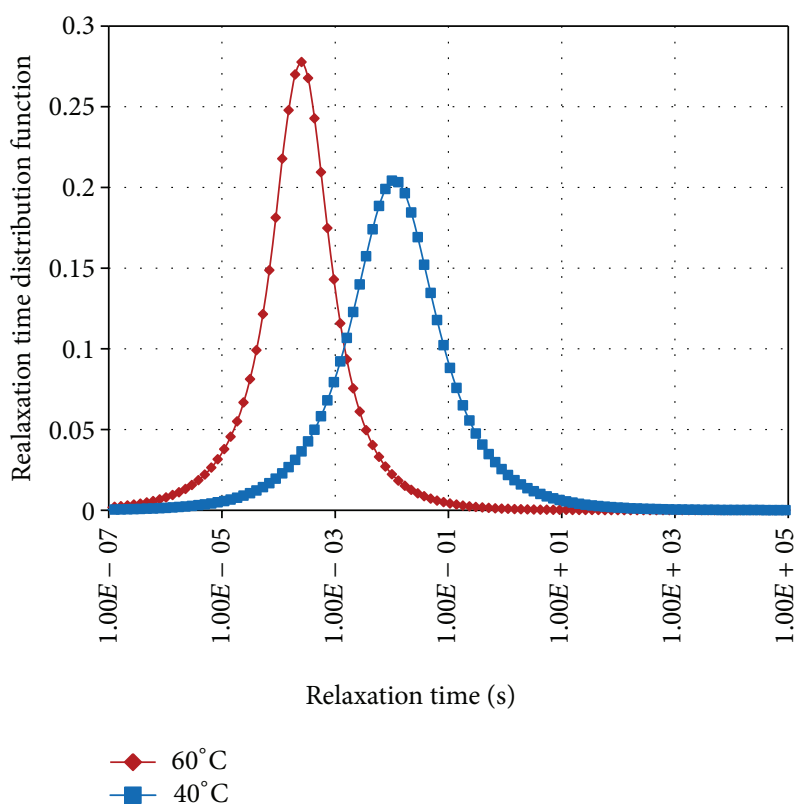

(b)

Figure 9: (a) Modeling of the dielectric response of a $87 / 10 / 3$ nanocomposite using (17) and (18); (b) corresponding relaxation time distribution for the interfacial peaks.

\subsection{Breakdown Strength of LLDPE/Clay Nanocomposites.} The calculated estimators $\widehat{\alpha}$ and $\widehat{\beta}$ of the Weibull parameters obtained from the statistical analysis of 13 different samples are given in Table 3 with the confidence bounds for the scale parameter (or at the 63.2 percentile). Since the breakdown strength, when measured using the ASTM short term test, is largely affected by the chosen procedure, such as rate of rise, type of electrodes, specimen thicknesses, and the type of surrounding medium, we must be extremely careful before assessing an improvement (or not) of this property for a particular material over another [30]. Accordingly, the breakdown measurements were conducted in two different

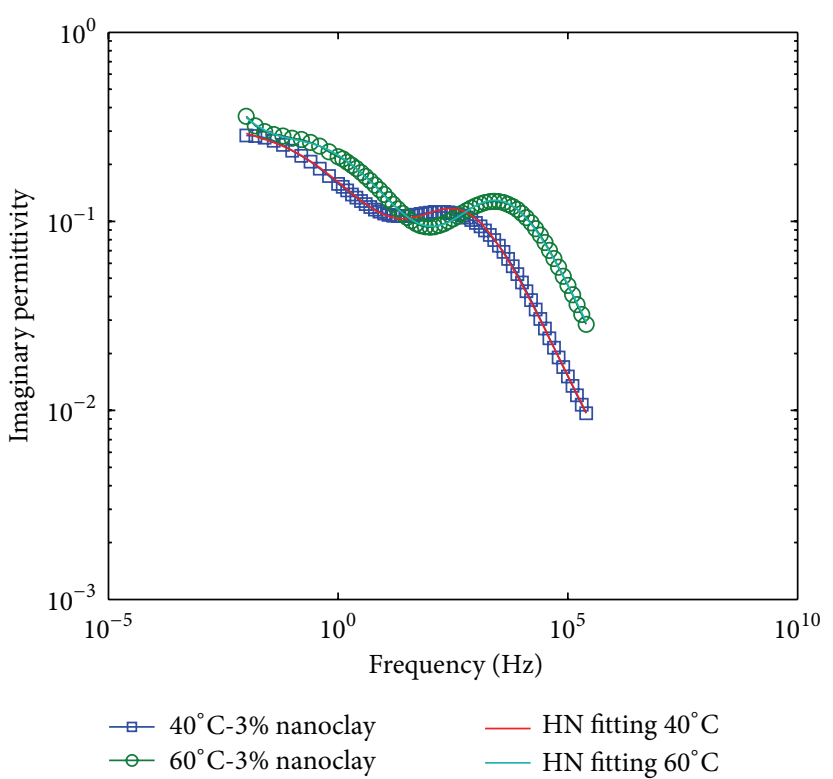

(a)

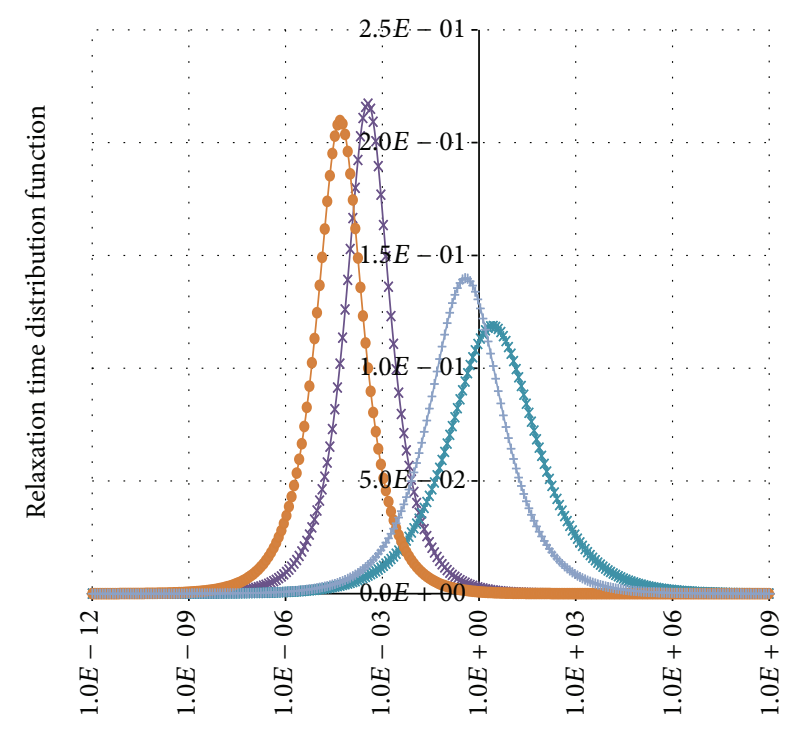

Relaxation time (s)

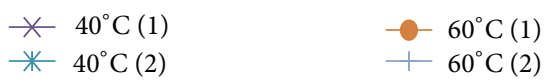

(b)

FIGURE 10: (a) Modeling of the dielectric response of a $97 / 0 / 3$ nanocomposite using (17) and (18) with two $\mathrm{H}-\mathrm{N}$ relaxation peaks; (b) corresponding relaxation time distribution for the interfacial peaks.

laboratories with the same equipment, using the same procedure described above. The measured breakdown strength was very consistent for the LLDPE, LLDPE/0/1, and LLDPE/0/3 samples between the two laboratories when the measurements were conducted on $0.59 \mathrm{~mm}$ specimens. Despite the poor dispersion of the clay platelets for the material without a compatibilizer, a notable improvement of the breakdown strength was observed following the incorporation of $3 \%$ of 


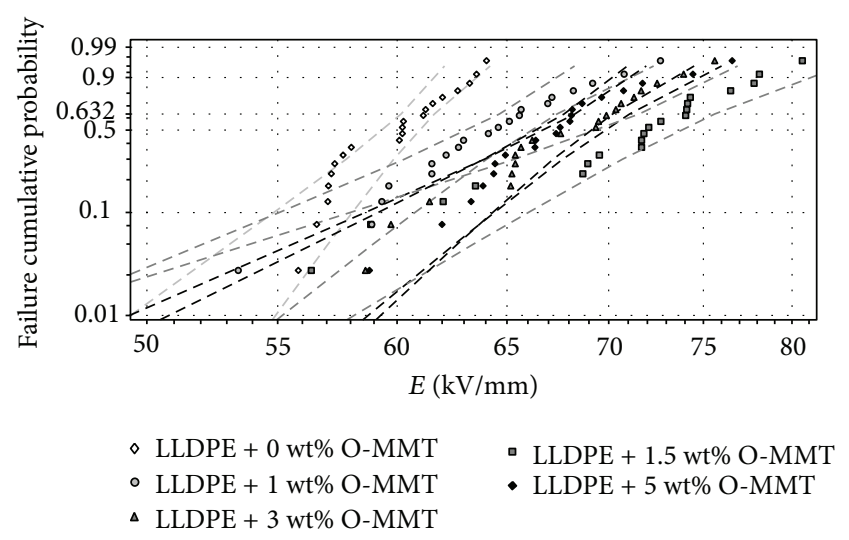

FIGURE 11: Weibull probability plot of dielectric strength of LLDPE with $0 \%, 1 \%$, and $3 \%$ wt of O-MMT with their $90 \%$ confidence intervals.

nanoclay, which is in good agreement with similar results previously published [28]. This is shown in Figure 11 in the form of a Weibull plot for the 0.59 samples measured in laboratory number 2. However, when the same procedure was repeated for a thinner specimen $(0.53 \mathrm{~mm})$, no improvement in the breakdown strength was observed. This can be explained by the inception of an intense partial discharge activity prior to breakdown, leading to a very fast erosion and failure of the tested specimens [30]. Another set of measurements were conducted on the same thin samples using different mineral oil with a higher dielectric strength, and a slight improvement of the dielectric strength was observed for the clay containing composites [30].

To investigate the relation between the microstructure and the breakdown strength of PE/nanoclay composites, the same measuring procedure was used for four new samples, LLDPE, LLDPE/0/1, LLDPE/0/3, and LLDPE/15/3, with a $0.61 \mathrm{~mm}$ thickness. The calculated estimators $\widehat{\alpha}$ and $\widehat{\beta}$ of the Weibull parameters for these four samples are given in Table 3, with the confidence bounds for the scale parameter, and also shown in the form of a Weibull plot in Figure 12 (without confidence bounds, for clarity). The same trend as what was observed previously was confirmed. In addition, it was clearly shown that an improvement of the exfoliation of the clay platelets leads to an improvement of the breakdown strength since the sample with the compatibilizer showed the highest breakdown strength.

\section{Conclusion}

A low level addition of nanoclay in a LLDPE matrix was found to significantly alter the material dielectric response, leading to a broad main interfacial relaxation peak. When a compatibilizer was used to improve the dispersion of the clay platelets, this peak was found to shift towards low frequencies. In addition, a second interfacial relaxation peak at a much lower frequency was observed for the samples with poor exfoliation, due to the anisotropy of the conductivity of clay tactoïds, which are much more conductive along the clay platelets than across. At power frequencies, the incorporation

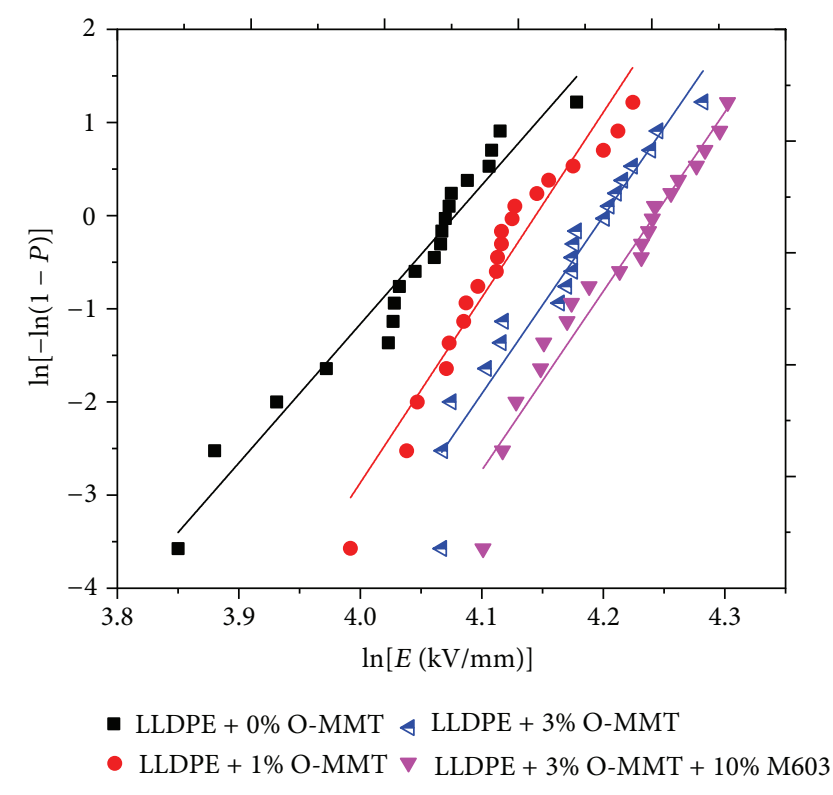

FIGURE 12: Weibull probability plot of the dielectric strength of pure LLDPE, LLDPE/0/1, LLDPE/0/3, and LLDPE/10/3.

of a small percentage of clay leads to an increase of roughly two orders of magnitude of the material dielectric losses. However, the dissipation factor of $\mathrm{PE} /$ clay nanocomposites still remains low enough that it does not have a real impact for its future use as an insulation wall for power cables.

The observed increase of the dielectric breakdown with the addition of nanoclay inside a PE matrix reported in this paper is similar to what has been reported previously [28]. It was also shown that the improvement of the clay exfoliation leads to an increase of the material dielectric breakdown strength.

\section{Acknowledgments}

The financial support of the Natural Sciences and Engineering Research Council of Canada (NSERC) and HydroQuébec is greatly acknowledged. C. Daran-Daneau would also like to thank Hydro-Québec and the Fonds de Recherche-Nature et Technologie (FRQNT) for their financial support. The authors would also like to acknowledge the assistance of Abdellah Ajji, Weawkamol Leelapornpisit, and Ahmad Zohre Vand from Department of Chemical Engineering of the École Polytechnique de Montréal for the sample preparation and the TEM micrographs.

\section{References}

[1] A. L. Sharma and A. K. Thakur, "Improvement in voltage, thermal, mechanical stability and ion transport properties in polymer-clay nanocomposites," Journal of Applied Polymer Science, vol. 118, no. 5, pp. 2743-2753, 2010.

[2] S. Mehta, F. M. Mirabella, K. Rufener, and A. Bafna, "Thermoplastic olefin/clay nanocomposites: morphology and mechanical properties," Journal of Applied Polymer Science, vol. 92, no. 2, pp. 928-936, 2004. 
[3] C. Trudel, A. D. Ngô, and T. Sean, "Low linear density polyethylene (LLDPE)/nanoclay composites: process parameters and mechanical properties," in Proceedings of the 8th Joint CanadaJapan Workshop on Composites, A. D. Ngô, J. Denault, A. Nakai, T. Morii, S. V. Hoa, and H. Hamada, Eds., pp. 112-123, DEStech Publications, Montréal, Canada, July 2010.

[4] K. Stoeffler, P. G. Lafleur, and J. Denault, "Effect of intercalating agents on clay dispersion and thermal properties in polyethylene/montmorillonite nanocomposites," Polymer Engineering and Science, vol. 48, no. 8, pp. 1449-1466, 2008.

[5] Nanocor, "P-802 nanomax polyolefin masterbatch product," http://www.nanocor.com/tech_sheets/P802.pdf.

[6] M. Fréchette, M. Trudeau, H. D. Alamdari, and S. Boily, "Introductory remarks on nanodielectrics," in Proceedings of the Annual Report Conference on Electrical Insulation and Dielectric Phenomena (CEIDP'01), pp. 92-99, Kitchener, Canada, October 2001.

[7] M. Fréchette and C. W. Reed, "On molecular dielectrics in their role in shaping and controlling nanodielectrics," in Proceedings of the Annual Report Conference on Electrical Insulation and Dielectric Phenomena (CEIDP '06), pp. 333-337, Kansas City, Mo, USA, October 2006.

[8] M. Fréchette, C. W. Reed, and H. Sedding, "Progress, understanding and challenges in the field of nanodielectrics," IEEJ Transactions on Fundamentals and Materials, vol. 126, no. 11, pp. 1031-1043, 2006.

[9] T. Tanaka and M. Fréchette, Eds., Polymer Nanocomposites: Fundamentals and Possible Applications to Power Sectors, CIGRÉ Technical Brochures, 2011.

[10] M. Fréchette, A. Allais, L. Kebbabi et al., "Progress in the field of nanodielectrics: potentiality for HV cable applications," in Proceedings of the 8th International Conference on Power Insulated Cables (Jicable '11), Versailles, France, June 2011.

[11] A. S. Vaughan, M. Fréchette, V. Englund, C. W. Reed, and T. Tanaka, "Strategies towards the development of novel nanostructured functional dielectrics," in Proceedings of the 7th Annual CIGRÉ Canada Conference on Power Systems, Montréal, Canada, September 2012.

[12] M. Fréchette, "Reflections on nanotechnology applied to dielectrics in a context of electrotechnical applications," in Proceedings of the SPE Polyolefins and Flexpack Conference, Houston, Tex, USA, February 2011.

[13] C. Calebrese, L. Hui, L. S. Schadler, and J. K. Nelson, "A review on the importance of nanocomposite processing to enhance electrical insulation," IEEE International Symposium on Electrical Insulation, vol. 18, no. 4, pp. 938-945, 2011.

[14] M. Fréchette, A. Vijh, L. Utracki et al., "Nanodielectrics: a panacea for solving all electrical insulation problems?" in Proceedings of the 10th IEEE International Conference on Solid Dielectrics (ICSD '10), pp. 130-158, Potsdam, Germany, July 2010.

[15] T. J. Lewis, "Interfaces are the dominant feature of dielectrics at the nanometric level," IEEE Transactions on Dielectrics and Electrical Insulation, vol. 11, no. 5, pp. 739-753, 2004.

[16] M. Fréchette, R. Y. Larocque, M. Trudeau et al., "Nanostructured polymer microcomposites: a distinct class of insulating materials," IEEE Transactions on Dielectrics and Electrical Insulation, vol. 15, no. 1, pp. 90-105, 2008.

[17] B. Zazoum, A. D. Ngô, E. David, C. Daneau, and M. Fréchette, "Morphology characteristics, thermal properties and dielectric breakdown of LLDPE/clay nanocomposites," in Proceedings of the 26th Technical Conference of the American Society for Composites and 2nd Joint Canadian-American International Conference on Composites, American Society for Composites, Montréal, Canada, 2011.

[18] A. K. Jonscher, Dielectric Relaxation in Solids, Chelsea Dielectrics Press, London, UK, 1983.

[19] A. Helgeson and U. Gafvert, "Dielectric response measurements in time and frequency domain on high voltage insulation with different response," in Proceedings of the International Symposium on Electrical Insulating Materials (ISEIM '98), pp. 393-398, September 1998.

[20] B. V. Hamon, "An approximate method for deducing dielectric loss factor from direct-current measurements," Proceedings of the IEE, vol. 99, pp. 151-155, 1952.

[21] M. J. Beran, Statistical Continuum Theories, Interscience Publishers, 1968.

[22] Y. P. Emets, "Electrical characteristics of three-component dielectric media," Journal of Experimental and Theoretical Physics, vol. 87, no. 3, pp. 612-620, 1998.

[23] E. Tuncer, S. M. Gubański, and B. Nettelblad, "Dielectric relaxation in dielectric mixtures: application of the finite element method and its comparison with dielectric mixture formulas," Journal of Applied Physics, vol. 89, no. 12, pp. 8092-8100, 2001.

[24] G. Bánhegyi, "Comparison of electrical mixture rules for composites," Colloid \& Polymer Science, vol. 264, no. 12, pp. 1030-1050, 1986.

[25] L. K. H. van Beek, "Dielectric behavior of heterogeneous systems," in Progress in Dielectrics, J. B. Birks, Ed., CRC Press, 1967.

[26] P. A. M. Steeman and J. van Turnhout, "Dielectric properties of inhomogeneous media," in Broadband Dielectric Spectroscopy, F. Kremer and A. Schönhals, Eds., Springer, 2003.

[27] S. Havriliak Jr. and S. J. Havriliak, Dielectric and Mechanical Relaxation in Materials: Analysis, Interpretation, and Application to Polymers, Hanser, Munich, Germany, 1997.

[28] C. D. Green, A. S. Vaughan, G. R. Mitchell, and T. Liu, "Structure property relationships in polyethylene/montmorillonite nanodielectrics," IEEE Transactions on Dielectrics and Electrical Insulation, vol. 15, no. 1, pp. 134-143, 2008.

[29] V. Tomer, G. Polizos, C. A. Randall, and E. Manias, "Polyethylene nanocomposite dielectrics: implications of nanofiller orientation on high field properties and energy storage," Journal of Applied Physics, vol. 109, no. 7, article 074113, pp. 1-11, 2011.

[30] C. Daran-Daneau, E. David, M. Fréchette, and S. Savoie, "Influence of the surrounding medium on the dielectric strength measurement of LLDPE/clay nanocomposites," in Proceedings of the IEEE International Symposium on Electrical Insulation (ISEI '12), pp. 654-658, San Juan, Puerto Rico, USA, June 2012. 

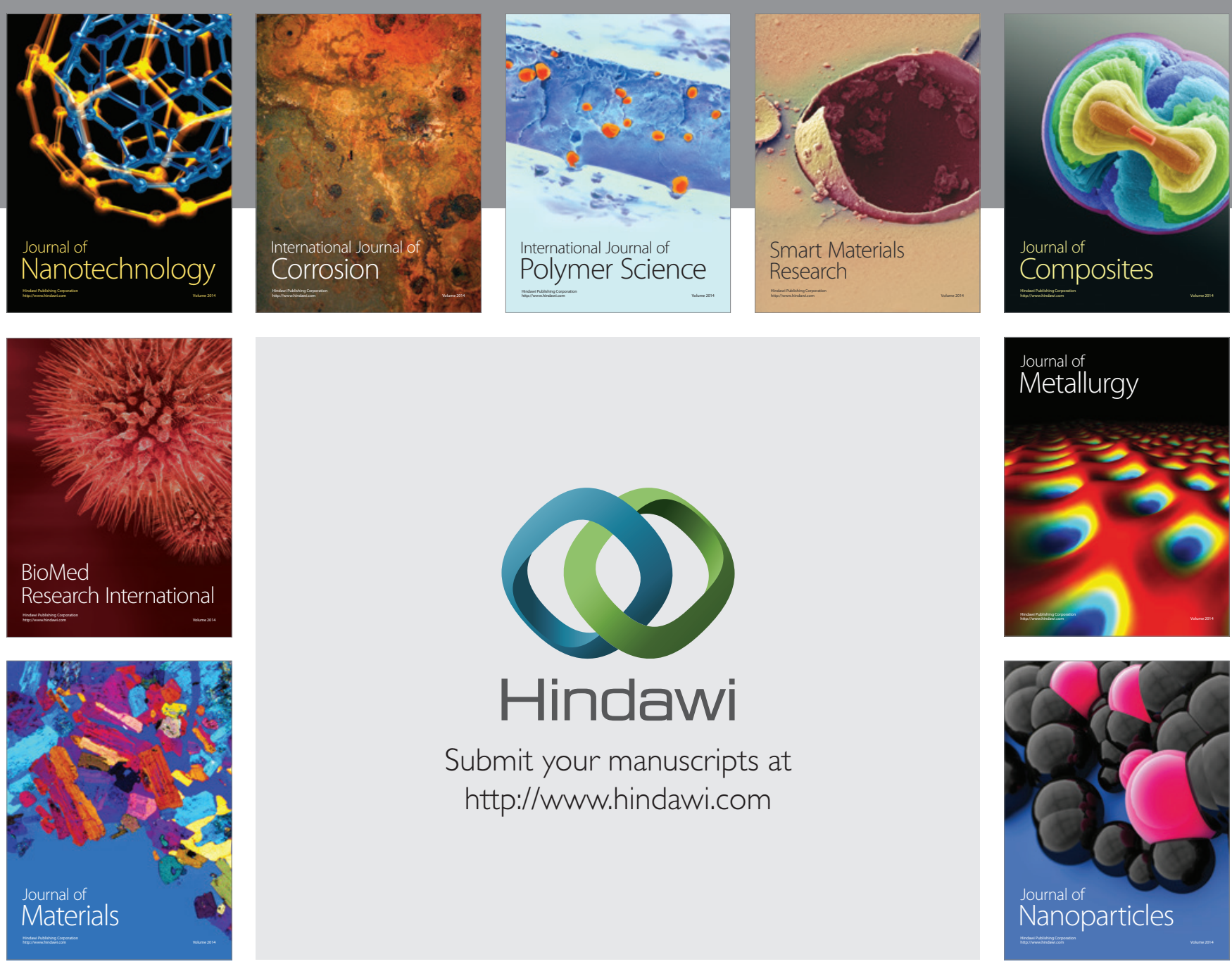

Submit your manuscripts at http://www.hindawi.com
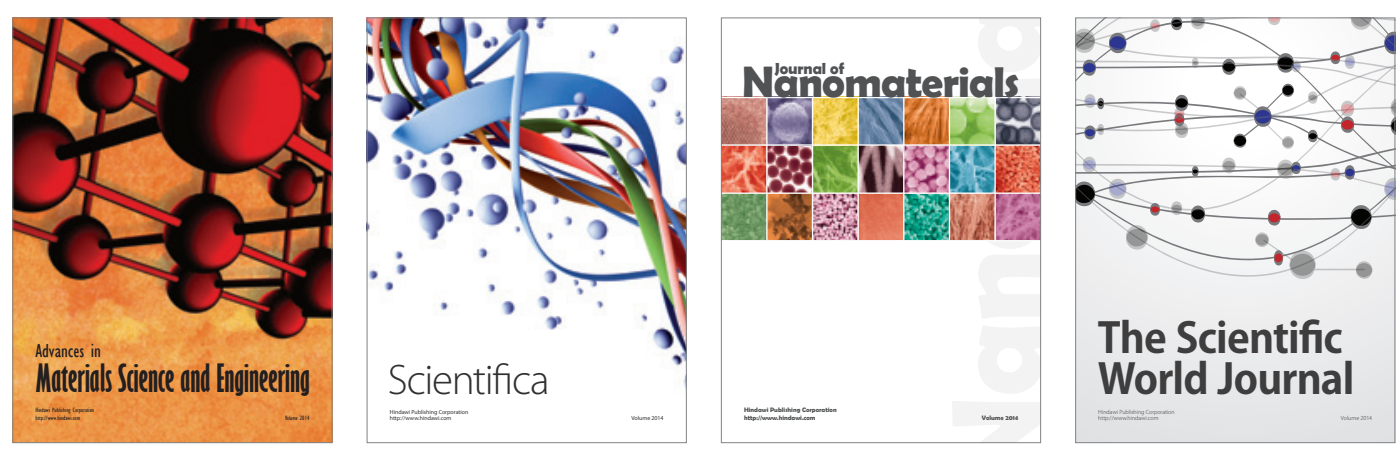

\section{The Scientific World Journal}
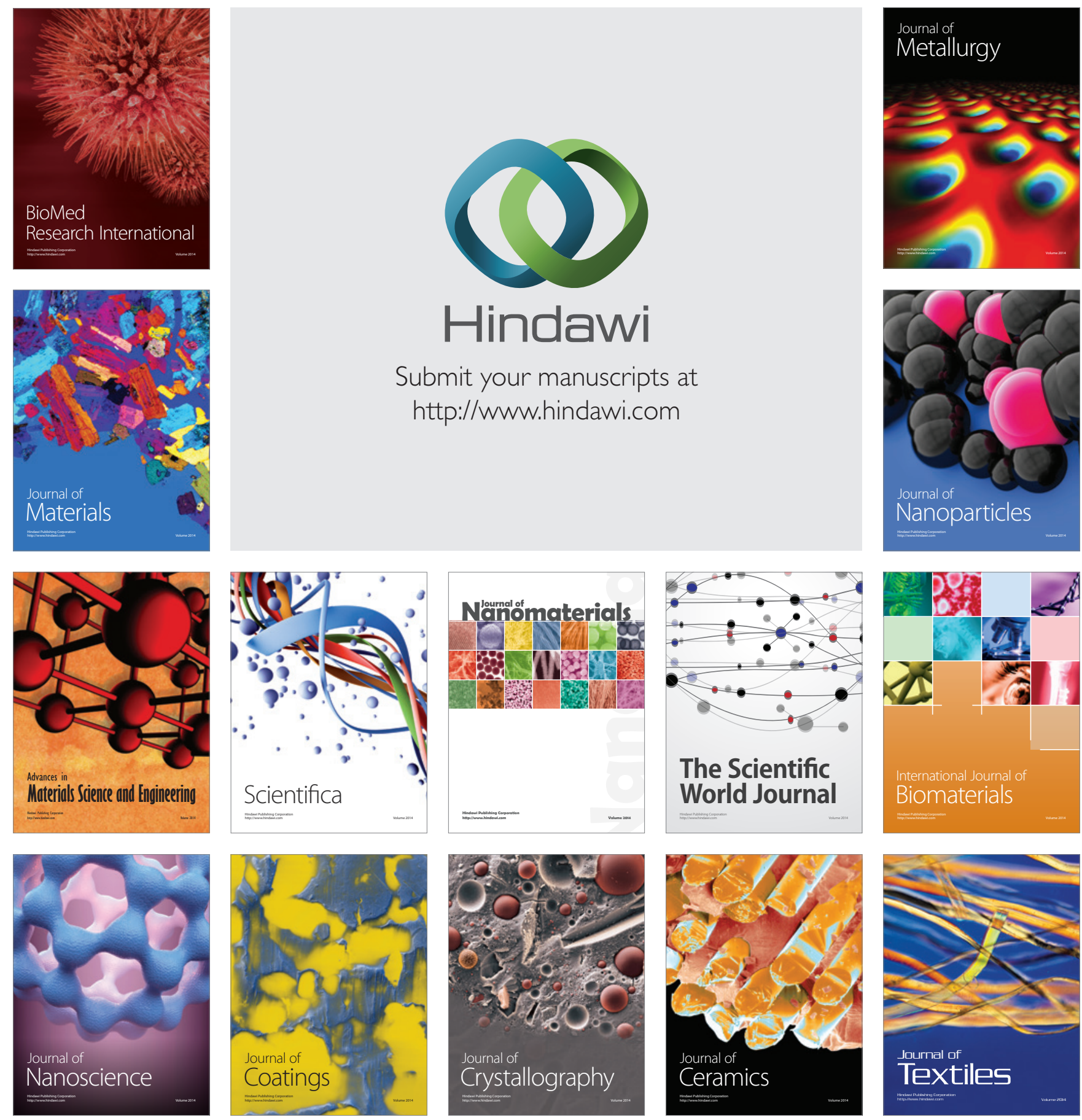